\title{
Avaliação psicológica e envelhecimento humano: revisão de pesquisas
}

\author{
Janaina Chnaider \\ Tatiana de Cássia Nakano
}

\section{RESUMO}

O envelhecimento humano tem sido foco de interesse da Psicologia nas últimas décadas, se tornando uma de suas áreas de atuação emergentes. Nesse sentido, o presente estudo contempla uma revisão sistemática da literatura sobre avaliação psicológica aplicada à população idosa brasileira. A busca nas bases de dados SciELO, Periódicos CAPES e PePSIC foi realizada com os descritores "avaliação psicológica e envelhecimento", "avaliação psicológica e idoso" e "avaliação psicológica e terceira idade", sem limitação de ano de publicação, resultou em 545 artigos. Após triagem e aplicação dos critérios de elegibilidade, 36 foram selecionados e analisados seguindo o método Prisma. Os resultados indicam que, de modo geral, as pesquisas ainda se mostram em número reduzido e bastante recentes, conduzidas principalmente junto a idosos que se encontram em instituições de saúde. As pesquisas se voltam, principalmente, à investigação de 21 diferentes construtos, relacionados, essencialmente, à depressão, a qualidade de vida e aos aspectos cognitivos, envolvendo, especialmente, os déficits e prejuízos associados ao envelhecimento. Pouca ênfase nos aspectos positivos e saudáveis foi encontrada nos estudos, de modo a corroborar que o envelhecimento ainda continua sendo visualizado como uma fase marcada por perdas e declínios, não tendo sido absorvida a concepção de envelhecimento bem-sucedido.

Palavras-chave: Velhice; Longevidade; Psicologia do idoso; Idosos.

\section{ABSTRACT}

\section{Psychological assessment and human aging: research review}

Aging has been a focus of interest in psychology in recent decades, becoming one of its emerging areas of expertise. In this sense, the present study contemplates a systematic review of the literature on psychological assessment applied to the elderly Brazilian population. The search in the SciELO, Periódico Capes and PePSIC databases was carried out with the descriptors "psychological assessment and aging," "psychological assessment and the elderly," and "psychological assessment and the elderly," without limitation of the year of publication, resulting in 545 articles. After screening and applying the eligibility criteria, 36 were selected and analyzed, following the Prisma method. The results indicate that, in general, research is still small and recent, conducted mainly with the elderly in health institutions. The research focuses on investigating 21 different constructs related to depression, quality of life, and cognitive aspects, involving, especially, the deficits and losses associated with aging. Little emphasis on positive and healthy aspects was found in the studies to corroborate that aging continues to be seen as a phase marked by losses and declines, and the concept of successful aging has not yet been absorbed.

Keywords: Old Age; Longevity; Developmental Psychology; Aging - Psychological Aspects; Aged.

0 aumento da expectativa de vida nos últimos 50 anos tem sido acompanhado por uma ampliação no interesse de pesquisadores de diferentes áreas sobre o processo de envelhecimento (Bartholomeus et al., 2019; Fernández-Ballesteros, 2009). 0 envelhecimento traz, consigo, uma série de desafios para a sociedade, especialmente relacionados a superação dos

\section{Sobre os autores \\ J. C. https://orcid.org/0000-0001- 5567-6698 \\ Pontifícia Universidade Católica de Campinas, Programa de Pós-Graduação stricto sensu em Psicologia - Campinas, SP Mestranda do programa de pós-graduação stricto sensu em Psicologia \\ Bolsista de Mestrado do CNPq jana_chnaider@hotmail.com \\ T. C. N. \\ http://orcid.org/0000-0002- 5720-8940 \\ Pontifícia Universidade Católica de Campinas, Programa de Pós- Graduação stricto sensu em Psicologia - Campinas, SP Docente do programa de pós- graduação stricto sensu em Psicologia \\ Doutora em Psicologia tatiananakano@hotmail.com}

\section{Direitos Autorais}

Este é um artigo de acesso aberto e pode ser reproduzido livremente, distribuído, transmitido ou modificado, por qualquer pessoa desde que usado sem fins comerciais. 0 trabalho é disponibilizado sob a licença Creative Commons CC-BY-NC.

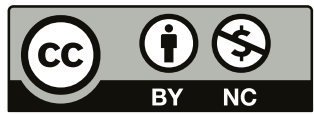




\section{M." INTERACÃO EM PSICOLOGIA}

estereótipos negativos que geralmente acompanham essa fase. Faz-se necessária a substituição por uma compreensão que abandone esses rótulos e se baseie na diversidade e contribuição que a população idosa pode trazer para a sociedade (Docking \& Stock, 2017).

A ideia principal é que o envelhecimento deixe de ser compreendido dentro de modelos em que predominam as noções de declínio e incapacidade (Fonseca, 2010), avançando para um modelo que visualiza a possibilidade de que esse processo possa ser vivenciado com satisfação, saúde, qualidade de vida e bem-estar (Lima et al., 2008), considerando-se os ajustamentos que ocorrem em diferentes aspectos: físico, social, emocional, econômico, profissional e cognitivo, dentre outros (Cupertino et al., 2007).

Nos últimos anos, uma série de pesquisas tem demonstrado que atitudes positivas perante o envelhecimento se mostram importantes preditores de funcionamento geral e bem-estar, manutenção da função cognitiva, diminuição dos níveis de depressão e ansiedade, bem como maior satisfação com a vida e envolvimento com comportamentos preventivos relacionados à saúde (Park \& Hess, 2019). Segundo Craciun et al. (2017), a valorização dos aspectos positivos da velhice pode ajudar a identificar recursos que facilitem o envelhecimento positivo, enquanto uma visão mais negativa pode atrapalhar os cuidados, os quais podem ser considerados desnecessários.

$\mathrm{Na}$ terceira idade, os indivíduos são confrontados com vários desafios, incluindo a transição para a aposentadoria, mais tempo livre, redução do contato social, além de outras mudanças no estilo de vida (Finkenzeller et al., 2019). Dentro desse contexto, os recursos psicológicos podem assumir um papel protetor durante esse processo adaptativo, destacando-se a importância de uma visão positiva sobre o envelhecimento, percepção de mais ganhos do que perdas, compreensão do futuro com uma expectativa positiva, assim como a continuidade no estabelecimento de metas na velhice (Craciun et al., 2017).

Consequentemente, os estudos voltados ao envelhecimento passam a voltar seu foco não apenas à manutenção da saúde, mas à maximização dos recursos psicológicos como autoeficácia, resiliência, satisfação com a vida, bem-estar (Hughes, 2016), longevidade, alta capacidade funcional, independência, adaptação positiva, hábitos saudáveis para redução de riscos, engajamento social, manutenção de altos níveis de habilidades funcionais e cognitivas (Teixeira \& Neri, 2008), abrindo um amplo campo de investigação para a Psicologia. Isso porque a análise integrada de várias dimensões psicológicas pode trazer compreensões importantes sobre o processo de envelhecimento, fornecendo base para a elaboração de intervenções voltadas à promoção de um envelhecimento saudável baseado na potencialização de recursos psicológicos (Ingrand et al., 2018).
Janaina Chnaider e Tatiana de Cássia Nakano
0 advento de uma perspectiva positiva em torno do envelhecimento vai ao encontro das premissas que embasam o movimento intitulado Psicologia Positiva, o qual tem, por objetivo principal, o estudo e o desenvolvimento de aspectos saudáveis no indivíduo (Pacico \& Bastianello, 2014), os quais poderiam possibilitar que o idoso consiga se organizar para empreender mudanças em seu estilo de vida, de modo a compreender de que a velhice pode ser uma etapa que permite prazer, conquistas e realizações (Kreuz \& Franco, 2017).

Esse conceito, chamado de envelhecimento positivo, envolve manter uma atitude positiva, sentir-se bem consigo mesmo, manter-se em forma e saudável e envolver-se plenamente na vida à medida que envelhece (Denmark \& Zarbiv, 2016). Dentro dessa compreensão, a identificação de aspectos positivos em pessoas com idade igual ou superior a 60 anos pode embasar a elaboração de políticas públicas que visem a criação de estratégias de intervenção e promoção de saúde a fim de garantir uma velhice saudável (Cavalcante et al., 2015; Ribeiro, 2015), possibilitando ainda a redução de gastos públicos com saúde, os quais, atualmente, representam um percentual elevado nas despesas governamentais (Veras, 2012).

Entretanto, mesmo frente a argumentos sólidos quanto à importância e necessidade de pesquisas que abordem os aspectos fortalecidos do ser humano no campo da psicologia, ainda se faz notar uma escassez de trabalhos (Pureza et al., 2012), especificamente em relação ao envelhecimento (Scorsolini-Comin \& Santos, 2010). Diante desse quadro, a revisão da literatura cientifica aqui proposta visa verificar se as pesquisas acompanharam essa mudança de paradigma sobre o envelhecimento, de modo a considerar que esse processo também pode estar associado a aspectos positivos (Dawalibi et al., 2013). Nesse sentido, a pesquisa teve, como objetivo principal, analisar a produção científica brasileira sobre avaliação psicológica aplicada à população idosa.

\section{MÉTODO}

Para atingir os objetivos propostos neste estudo, uma busca nas bases de dados eletrônicas da Coordenação de Aperfeiçoamento de Pessoal de Nível Superior (CAPES), Periódicos Eletrônicos de Psicologia (PePSIC) e Scientific Eletronic Library Online (SciELO) foi realizada. O estudo seguiu as recomendações propostas pelo método PRISMA-P (Moher et al., 2015).

\section{ESTRATÉGIA DE BUSCA}

As bases de dados eletrônicas foram consultadas em agosto de 2020, buscando-se por trabalhos que tivessem, como tema, a avaliação psicológica em idosos. Foram uti- 


\section{n* INTERACÃO EM L* PSICOLOGIA}

lizados três operadores boleanos: "avaliação psicológica e envelhecimento", "avaliação psicológica e idoso" e "avaliação psicológica e terceira idade" separadamente, de maneira que as autoras optaram pela utilização das terminologias mais comumente utilizadas para descrever essa população. Não houve limitação de ano de publicação.

\section{CRITÉRIOS DE ELEGIBILIDADE}

Foram aplicados os seguintes critérios de elegibilidade: trabalhos empíricos que utilizaram avaliação psicológica com foco no idoso (população com idade igual ou superior a 60 anos), sendo excluídos aqueles que, após a leitura dos resumos, encontravam-se duplicados, não abordavam a temática pesquisada, tivessem sido realizados em população estrangeira, trabalhos teóricos, artigos de revisão, resenhas, dissertações e teses sobre a temática. Os resumos foram avaliados por dois revisores, de forma independente.

\section{REGISTRO DE ESTUDOS}

Os registros foram feitos em uma planilha no programa Excel. A partir da leitura dos resumos dos artigos, por dois revisores independentes, uma triagem dos trabalhos foi realizada. Foram selecionados aqueles que atendiam ao foco da revisão sistemática. Posteriormente, os mesmos revisores atuaram nas demais fases, de elegibilidade, inclusão e análise.

\section{EXTRAÇÃO DOS DADOS}

Posteriormente, após a seleção dos trabalhos, optou-se pela análise das seguintes categorias: ano de publicação, local de origem da amostra, sexo dos participantes, área de conhecimento dos autores, construtos avaliados e instrumentos utilizados. Nos casos em que um trabalho contemplava mais de uma categoria, a classificação foi feita em todas as categorias apresentadas no artigo, de modo que, em algumas delas, o número total de classificações ultrapassa o número de artigos analisados.

Os dados foram analisados estimando-se a frequência e porcentagem de ocorrência de cada categoria de resposta.

\section{AVALIAÇÃO DO RISCO DE VIÉS}

A seleção das bases de dados, bem como a limitação de acesso a sua versão eletrônica, pode ter atuado de modo a impedir que outros artigos não tenham sido localizados na busca realizada. Do mesmo modo a seleção dos operadores boleanos e a impossibilidade de acesso a alguns textos completos também devem ser citados como possíveis vieses da revisão.

\section{RESULTADOS E DISCUSSÃO}

O percurso metodológico empregado na seleção dos artigos, segundo o método Prisma (Moher et al., 2009), é apresentado na Figura 1. Após tal procedimento, um total de 36 artigos foram selecionados, sendo 16 provenientes da base Periódicos CAPES, oito da PePSIC e 12 da SciELO.

Como forma de oferecer um detalhamento acerca do percurso de seleção dos artigos, a Figura 2 apresenta os resultados considerando-se os operadores boleanos e base de dados.

O primeiro dado analisado apontou que o primeiro trabaIho identificado foi publicado em 2006 e, o último, em 2021. 0 maior número de artigos foi encontrado nos anos de 2010 , 2013 e 2017 . Em contrapartida, nenhum estudo foi publicado nos anos de 2008, 2014 e 2020.

Se considerarmos que, durante a busca, não houve limitação de período e que foram encontrados artigos somente nos últimos 16 anos, poderemos ver que uma média de 2,25 artigos por ano foram publicados na temática, valor bastante reduzido perante a importância do assunto.

Diante da oscilação presente em relação a análise das publicações por ano, não se pode afirmar que houve o despertar do interesse da área da avaliação psicológica para essa fase do desenvolvimento. 0 número reduzido de trabalhos aponta para o fato de que, indiferente ao envelhecimento populacional, aumento da longevidade e transição demográfica da população, bem como a nova demanda por serviços de financiamento, suporte e políticas públicas destinadas à população idosa, tal temática ainda tem sido pouco investigada, notadamente em relação aos aspectos positivos que podem decorrer dessa fase, dentro do conceito de envelhecimento bem-sucedido (Kreuz \& Franco, 2017).

Em seguida, o local de origem da amostra, ou seja, onde os idosos foram recrutados, indicou quatro categorias: instituições de saúde ( $n=23 ; 63,8 \%$ ), grupos específicos de terceira idade ( $n=7 ; 19,4 \%)$, comunidade $(n=2 ; 5,5 \%)$ e instituições de longa permanência $(n=1 ; 2,7 \%)$. Ainda no que se refere à origem dos participantes, aqueles originários de instituições de saúde, como Unidades Básicas de Saúde (UBSs), hospitais e programas de apoio à saúde realizados por Universidades e Programas de Estratégias de Saúde desenvolvidos pelo setor público corresponderam a maior parte da amostra. É importante citar que o número da frequência apresentada na Tabela é maior do que o número de estudos analisados, visto que alguns estudos apresentaram mais de um local de recrutamento dos participantes. Também se destaca o fato de que parte das pesquisas $(n=3 ; 10,3 \%)$ não informaram o local onde os participantes foram localizados. 


\section{W'INTERACÃO EM ETS PSICOLOGIA}

Janaina Chnaider e Tatiana de Cássia Nakano

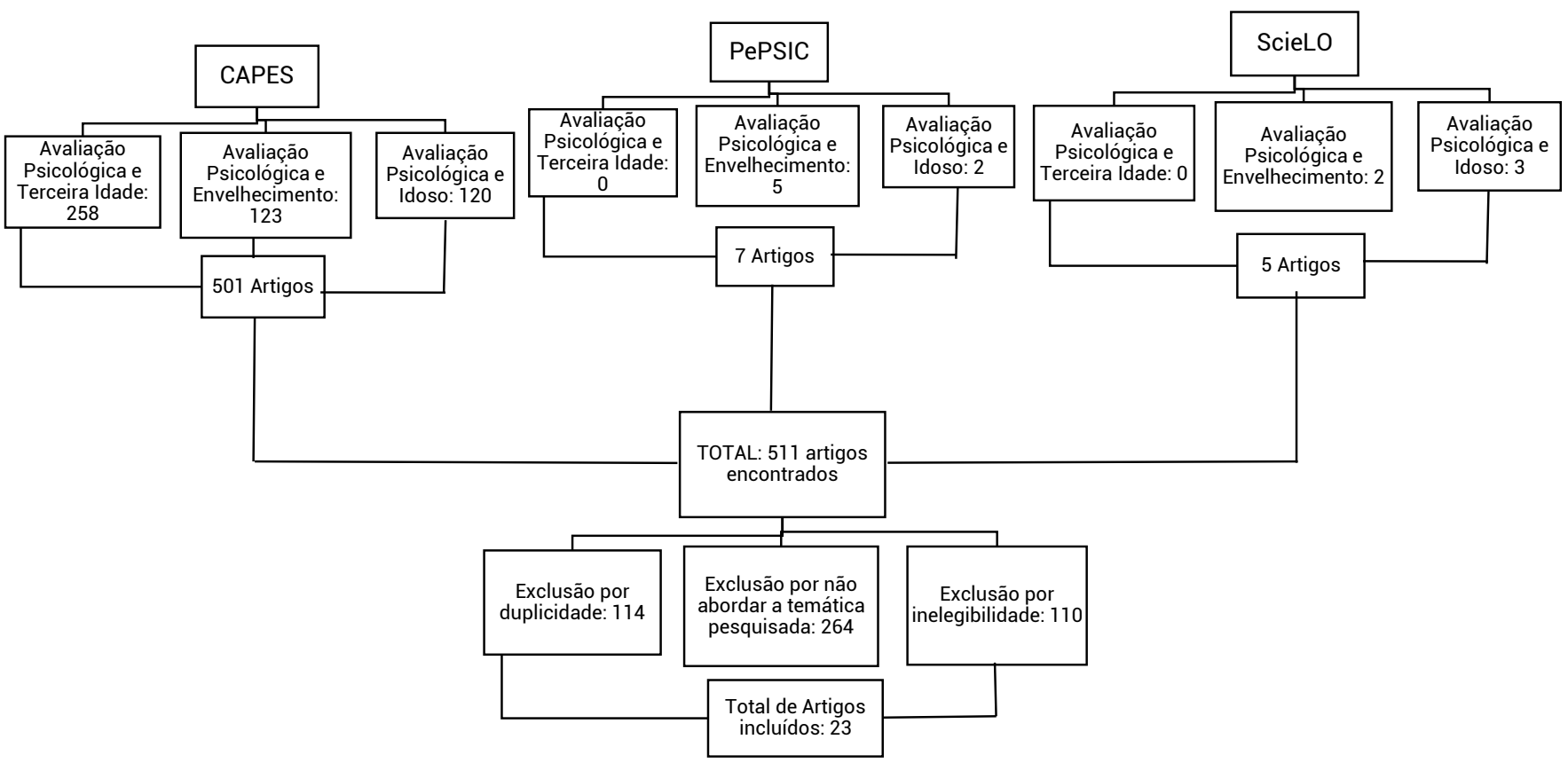

Figura 1. Fluxograma de Busca e Seleção dos Artigos

\begin{tabular}{cccc}
\hline Base de dados / operadores boleanos & Capes & Pepsic & Scielo \\
\hline Avaliação psicológica e terceira idade & 258 & 0 & 3 \\
\hline Avaliação psicológica e envelhecimento & 123 & 8 & 14 \\
\hline Avaliação psicológica e idoso & 120 & 2 & 17 \\
\hline Total & 501 & 10 & 34 \\
\hline Selecionados & 16 & 8 & 12 \\
\hline
\end{tabular}

Figura 2. Percurso de Seleção dos Artigos, Considerando-se as Bases de Dados e Operadores Boleanos

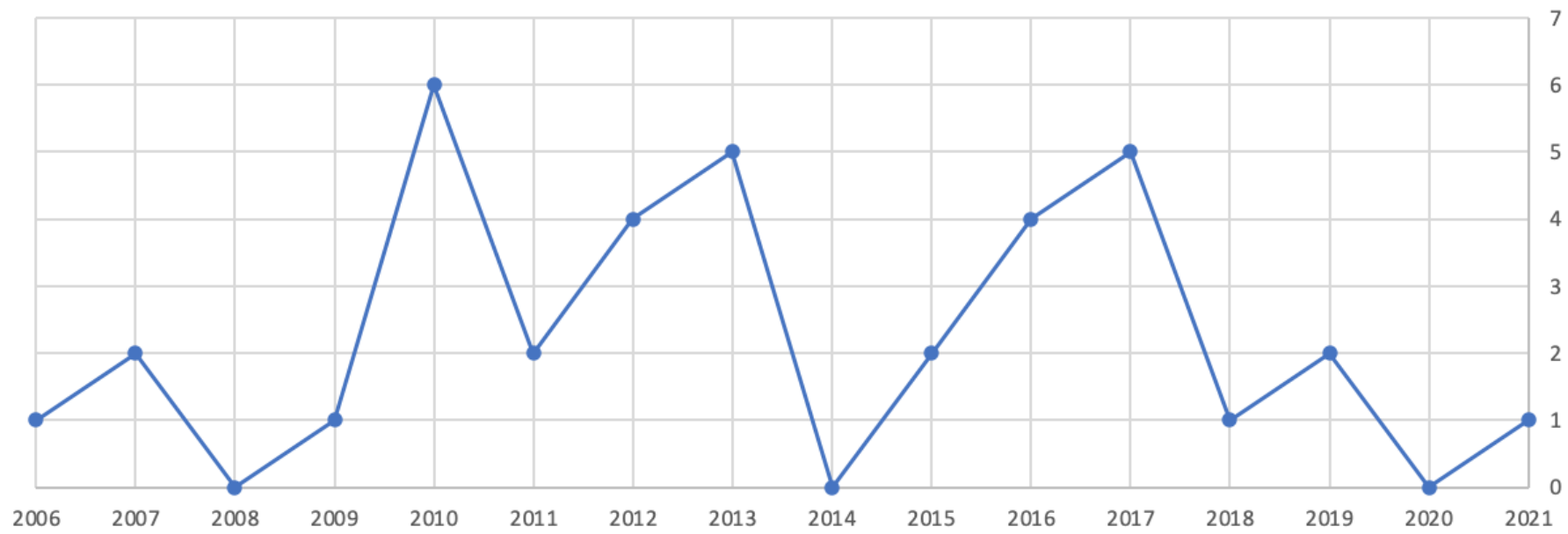

Figura 3. Número de Artigos por Ano de Publicação

Nota. Eixo horizontal $=$ ano de publicação; eixo vertical $=$ número de artigos publicados 


\section{-4: INTERACÃO EM LF PSICOLOGIA}

A predominância de idosos recrutados em instituições de saúde, pode indicar, por um lado, certo interesse ou preocupação com o bem-estar físico e mental, mas, por outro, este resultado pode refletir a ausência de programas de prevenção, de modo que a alta busca por serviços de saúde pode estar relacionada ao adoecimento e perdas resultantes do processo de envelhecimento (Alberte et al., 2015; Schneider \& Irigaray, 2008). Em contrapartida, as pesquisas com idosos originários de Grupos de Terceira Idade evidência a aderência dessa população a estes espaços. A participação de idosos em grupos que oferecem atividades desenvolvidas especificamente para adultos mais velhos favorecem tanto a saúde física e mental, contribuindo, consequentemente, para o alcance da velhice bem-sucedida, além de favorecer a quebra do estereótipo da pessoa idosa como aquele indivíduo desocupado (Cavalcante et al., 2015). Assim, supõe-se que, além da conveniência em encontrar participantes, as pesquisas que tiveram população proveniente de Grupos de Terceira Idade podem apresentar os benefícios que esses espaços e ações podem proporcionar.

Em relação à amostra dos estudos, o sexo dos participantes também foi investigado. Os resultados mostraram que em $94,3 \%$ dos estudos a população foi constituída por homens e mulheres, ainda que, na maior parte das pesquisas (83,4\%), o número de participantes do sexo feminino tenha sido superior ao de participantes do sexo masculino. Três estudos não apresentaram a descrição do sexo dos participantes.

Ao observar essas informações, pode-se supor que a predominância de idosas nos estudos encontrados esteja relacionado com os locais de coleta, visto que as mulheres tendem a procurar mais ajuda tanto nos serviços referentes a saúde quanto nos serviços que oferecem suporte social (Freire et al., 2015). Esses resultados também podem ser associados aos índices de mortalidade de idosos do sexo masculino, superior ao feminino, de modo a confirmar a presença de aspectos culturais, que resultam na negligência do homem em relação à sua saúde (Freire et al., 2015).

0 dado analisado a seguir buscou identificar a área de conhecimento que os pesquisadores pertenciam. Para isso, a formação de cada autor, indicada em cada artigo, foi levantada. Conforme esperado, dado o foco na área da avaliação psicológica, foi identificada predominância de autores com formação em Psicologia ( $n=34 ; 40,0 \%$ ). Considerando-se que a área específica investigada, no caso, a avaliação psicológica, a presença predominante desse profissional se justifica. Isso porque o uso de instrumentos psicológicos é restrito a esse profissional de maneira que o emprego desse tipo de instrumento em pesquisas realizadas por outras áreas de conhecimento deve envolver um trabalho multidisciplinar, que garanta a presença de um psicólogo responsável.
Janaina Chnaider e

Tatiana de Cássia Nakano
Número importante de estudos também foram conduzidos por profissionais da área de Enfermagem ( $n=25 ; 29,4 \%$, Medicina ( $n=13 ; 15,3 \%)$, Fisioterapia, Odontologia, Fonoaudiologia e Educação Física ( $n=2 ; 2,3 \%$ cada). Foram encontrados pesquisadores de outras áreas, nas com apenas um artigo em cada (Ciências Biológicas, Engenharia Elétrica, Farmácia Bioquímica, Nutrição, Educação Física e Psicomotricidade). Essa diversidade de áreas de atuação dos pesquisadores reforça o caráter multiprofissional de alguns eixos de estudo da Psicologia Positiva.

Vale ressaltar que um número bastante elevado de profissionais pertencentes a nove outras áreas de conhecimento áreas foi encontrado como autores dos trabalhos analisados $(60,0 \%)$. Este resultado corrobora os resultados apresentados por Pires, Nunes e Nunes (2015), segundo os quais, tem se feito notar a atração de profissionais de outras áreas pela avaliação de construtos positivos e o impacto desses aspectos na vida dos indivíduos.

O próximo dado analisado envolveu o levantamento dos construtos avaliados nos artigos. Um total de 21 diferentes foram encontrados, sendo importante destacar que algumas pesquisas envolveram mais de um construto, de modo que o número total de classificações $(n=58)$ ultrapassa o número de artigos analisados $(n=36)$.

Tabela 1. Construtos Investigados nas Pesquisas

\begin{tabular}{ccc}
\hline Construto & Frequência & $\%$ \\
\hline Depressão & 5 & 15 \\
Qualidade de Vida & 5 & 15 \\
Cognição & 3 & 8,8 \\
Memória & 3 & 8,8 \\
Autopercepção do Envelhecimento & 2 & 5,9 \\
Funções Executivas & 2 & 5,9 \\
Interação Social & 2 & 5,9 \\
Ansiedade & 1 & 2,9 \\
Autoestima & 1 & 2,9 \\
Capacidade funcional & 1 & 2,9 \\
Coping & 1 & 2,9 \\
Criatividade & 1 & 2,9 \\
Espiritualidade & 1 & 2,9 \\
Habilidades Sociais & 1 & 2,9 \\
Inteligência & 1 & 2,9 \\
Relações Interpessoais & 1 & 2,9 \\
Satisfação com a Vida & 1 & 2,9 \\
Suporte Familiar & 1 & 2,9 \\
Suporte Social & 1 & 2,9 \\
\hline Total & 34 & 100 \\
\hline
\end{tabular}




\section{MLE INTERACÃO EM L* PSICOLOGIA}

Dos 21 construtos investigados nas pesquisas analisadas, pode-se ver que a maior parte deles envolve aspectos positivos, com exceção de depressão, estresse e ansiedade. Convém destacar, no entanto, que os demais construtos, apesar de mostrarem, inicialmente, um caráter positivo, comumente foram investigados em relação a prejuízos ou déficits nessa fase, por exemplo, em relação a qualidade de vida, cognição, memória, suporte social e familiar, dentre outros. Nesse sentido, recomenda-se certa cautela ao afirmar que os estudos parecem focar-se em aspectos positivos relacionados ao envelhecimento.

Entretanto, a presença de diversos construtos que são englobados na psicologia positiva (qualidade de vida, autoestima, coping, criatividade, espiritualidade, relações interpessoais, satisfação com a vida) parece confirmar um interesse pelo envelhecimento dentro de um modelo que busca valorizar as forças presentes nos indivíduos nessa idade.

Tais dados confirmam a preocupação com a manutenção de uma visão positiva, a qual tem sido associada a maiores níveis de satisfação com a vida, autoavaliação de saúde, ampliação das redes de contato sociais, bem-estar, atuando como recurso protetivo ao processo de envelhecimento (Bellingtier \& Neupert, 2018). No entanto, a percepção de ausência de outros construtos, tais como bem-estar, satisfação com a vida, autoeficácia, percepção de saúde, confirmam a opinião apresentada por Finkenzeller et al. (2019), de que poucos estudos têm examinado o envelhecimento sob uma perspectiva abrangente, que considere construtos psicológicos que influenciam, positivamente, a qualidade de vida dos idosos.

Em seguida, os instrumentos utilizados nas pesquisas foram listados, de acordo com o construto avaliado. Um total de 51 instrumentos foram encontrados, agrupados em treze principais focos (Tabela 2). Convém ressaltar que a avaliação psicológica de idosos se mostra bastante desafiadora dada a escassez de instrumentos psicométricos desenvolvidos para tal faixa etária (Edelstein et al., 2007), de modo que a maior parte dos instrumentos não são específicos para idosos, abrangendo, geralmente, adultos.

Dentre os construtos mais pesquisados na população idosa destacam-se os aspectos cognitivos e inteligência (31,4\%), por meio de 14 diferentes instrumentos. Dentre eles, o WAIS-III e o Mini Exame do Estado Mental se destacam. O interesse pelo construto nessa faixa etária, baseia-se no fato de que, em geral, a literatura tem demonstrado que o declínio cognitivo ocorre como um aspecto normal do envelhecimento, sendo que, até os 75 anos, as mudanças cognitivas se mostram, comumente, mais sutis ou inexistentes (Argimon, 2006). Entretanto, diferentes níveis de comprometimentos cognitivos podem se fazer presentes nos idosos, envolvendo desde aqueles que conseguem adquirir novas informações e habilidades, sendo capazes de lembrar e usar bem as habilidades que já dominam, como outros em que as alterações do estado cognitivo e a não realização de atividades antes exercidas se mostram bastante intensificadas, sendo esses um dos principais motivos da institucionalização dos idosos (Mello et al., 2012).

Em seguida, são encontrados os estudos voltados à investigação da depressão (14,0\%), notando-se predominância no uso da Geriatric Depression Scale (GDS). A grande quantidade de pesquisas voltada a avaliação desse construto pode ser compreendida perante a constatação de que, aproximadamente $4,4 \%$ dos idosos atingem os critérios para o diagnóstico de transtorno depressivo (Edelstein et al., 2007).

Nessa idade, uma série de estereótipos vêm sendo associados à velhice, como sofrimento, inatividade, dependência de outros, declínio da saúde, dor, incapacidade (Lopes et al., 2010), dificultando a adaptação à velhice, bem como a preparação para essa fase. Consequentemente, o surgimento de quadros relacionados à depressão pode ocorrer, decorrentes da interpretação que o idoso faz dos declínios característicos do envelhecimento (Lima et al., 2016; Papália \& Feldman, 2012), aumentando sua prevalência quando diante de acontecimentos de vida que se traduzem em perdas ou quando sua capacidade de adaptação se mostra diminuída (Azeredo \& Afonso, 2016). A associação entre esses sentimentos pode desencadear uma síndrome depressiva, comum em idosos, principalmente os institucionalizados.

Os aspectos neuropsicológicos e funções executivas foram investigados em 17,4\% dos estudos, envolvendo aspectos como memória, construção visuo-espacial, flexibilidade cognitiva, orientação espacial, atenção, percepção visual, linguagem, controle inibitório, velocidade verbal, dentre outros. Segundo Foss et al. (2010), as explicações sobre o envelhecimento estariam incompletas sem a inclusão das funções executivas, ainda mais se considerarmos que a perda de memória é uma das queixas mais presentes na terceira idade. Tal fato justifica a relevância, segundo os autores, de se investigar os processos atencionais, funcionamento executivo, memória episódica e conteúdos visuo-espaciais. $O$ instrumento mais utilizado para essa finalidade foi o Teste Wisconsin de Classificação de Cartas.

Um número importante de publicações avalia a qualidade de vida (14,0\%), por meio de oito diferentes instrumentos, sendo predominante o WHOQOL Brief. Esse construto vem sendo avaliado sob a perspectiva do próprio idoso e cujos resultados vêm sendo utilizados como parâmetro de avaliação de políticas, intervenções de saúde e avaliações do cuidado a ser prestado, para além do enfoque na doença (Paskulin et al., 2010). Nessa população, a análise da qualidade de vida busca compreender como eles vivem e obter informações que permitam avaliar seu nível de satisfação de acordo com suas necessidades, bem como a identificação das melhorias que possam auxiliá-lo a manter um nível adequado de qualidade de vida (Esteve-Clavero et al., 2018). 


\section{W MTERAC̄̄oEM PSICOLOGIA}

Tabela 2. Instrumentos Utilizados nas Pesquisas

\begin{tabular}{|c|c|c|c|c|}
\hline Construto avaliado & Autores & Instrumento & $n$ & $\%$ \\
\hline \multirow{14}{*}{$\begin{array}{c}\text { Cognitivo } \\
\text { /inteligência } \\
\quad(n=27)\end{array}$} & $\begin{array}{l}\text { Lopes e Argimon (2009); Netto et al. } \\
\text { (2013); Soares et al. (2013) }\end{array}$ & Wechsler Adult Intelligence Scale-III (WAIS-III) & 3 & 5,8 \\
\hline & Soares et al. (2013) & Bateria de Breve Rastreio Cognitivo (BBRC) & 1 & 1,9 \\
\hline & Souza e Wechsler (2013) & Bateria de Habilidades Intelectuais de Adultos (BAID) & 1 & 1,9 \\
\hline & Oliveira et al. (2017) & Escala de memória Wechsler revisada (WMS-R) & 1 & 1,9 \\
\hline & Gurgel e Sisto (2010) & Teste Pictórico de Memória & 1 & 1,9 \\
\hline & Cecato et al. (2013) & Elder Memory Test & 1 & 1,9 \\
\hline & Santos et al. (2021) & Rivermead Behavioral Memory Test & 1 & 1,9 \\
\hline & Santos et al. (2021); Soares et al. (2013) & Teste de Fluência Verbal & 2 & 3,9 \\
\hline & Netto et al. (2013) & Teste de Stroop de cores e palavras & 1 & 1,9 \\
\hline & Souza et al. (2007) & $\begin{array}{l}\text { Questionnaire on Cognitive Decline in the elderly } \\
\text { (IQCODE) }\end{array}$ & 1 & 1,9 \\
\hline & Oliveira et al. (2019) & Addenbrooke;s cognitive examination revised & 1 & 1,9 \\
\hline & $\begin{array}{l}\text { Apratto Júnior (2010); Esteves et al. (2016); } \\
\text { Foss et al. (2010); Lampert e Scortegagna } \\
\text { (2017); Lopes e Argimon (2009); Netto et al. } \\
\text { (2013); Reis et al. (2011); Rien et al. (2017); } \\
\text { Santos et al. (2021); Soares et al. (2013); } \\
\text { Souza et al. (2007) }\end{array}$ & Mini Exame do Estado Mental (MEEM) & $\begin{array}{l}1 \\
1\end{array}$ & $\begin{array}{c}21 \\
6\end{array}$ \\
\hline & Esteves et al. (2016) & WCST-64 (versão abreviada de 64 cartas) & 1 & 1,9 \\
\hline & Gurgel e Sisto (2010) & Teste de Raciocínio Inferencial (RIn) & 1 & 1,9 \\
\hline \multirow{2}{*}{$\begin{array}{l}\text { Autopercepção } \\
(\mathrm{n}=2)\end{array}$} & Rien et al. (2017) & Sistema Compreensivo de Zulliger (ZCS) & 1 & 1,9 \\
\hline & Lampert e Scortegagna (2019) & Rorschach - sistema compreensivo & 1 & 1,9 \\
\hline Criatividade $(n=1)$ & Souza e Wechsler (2013) & Pensando Criativamente com Palavras e Figuras & 1 & 1,9 \\
\hline \multirow[t]{3}{*}{ Depressão $(n=12)$} & $\begin{array}{l}\text { Baptista et al. (2006); Brito et al. (2012); } \\
\text { Carneiro e Falcone (2016); Esteves et al. } \\
\text { (2016); Lampert e Scortegagna (2017); } \\
\text { Lopes e Argimon (2009); Netto et al. (2013); } \\
\text { Oliveira et al. (2019); Soares et al. (2013) }\end{array}$ & Geriatric Depression Scale (GDS) & 9 & $\begin{array}{c}17 \\
6\end{array}$ \\
\hline & Brito et al. (2012); Lopes e Argimon (2009) & Inventário de Ansiedade de Beck (BAI) & 2 & 3,9 \\
\hline & Lampert e Scortegagna (2017) & Escala de Pensamentos Depressivos (EPD) & 1 & 1,9 \\
\hline $\begin{array}{c}\text { Ansiedade } \\
(\mathrm{n}=3)\end{array}$ & $\begin{array}{l}\text { Bastos et al. (2016); Brito et al. (2012); } \\
\text { Lopes e Argimon (2009) }\end{array}$ & Inventário de Depressão de Beck (BDI) & 3 & 5,9 \\
\hline \multirow{11}{*}{$\begin{array}{c}\text { Aspectos } \\
\text { neuropsicológicos } \\
\text { e/ou funções } \\
\text { executivas }(n=15)\end{array}$} & $\begin{array}{l}\text { Brito et al. (2012); Esteves et al. (2016); Lopes } \\
\text { e Argimon (2009); Silva-Filho et al. (2007) }\end{array}$ & Teste Wisconsin de Classificação de Cartas (WCST) & 4 & 7,8 \\
\hline & Foss et al. (2010) & Figuras complexas de Rey (FCR) & 1 & 1,9 \\
\hline & Foss et al. (2010) & Figuras complexas de Rey para idosos & 1 & 1,9 \\
\hline & Netto et al. (2013) & $\begin{array}{l}\text { Bateria Montreal de Avaliação da } \\
\text { Comunicação (Bateria MAC) }\end{array}$ & 1 & 1,9 \\
\hline & Santos et al. (2021); Soares et al. (2013) & Teste de Trilhas & 2 & 1,9 \\
\hline & Netto et al. (2013) & Teste de aprendizagem auditiva-verbal de Rey & 1 & 1,9 \\
\hline & Netto et al. (2013) & Teste Hayling & 1 & 1,9 \\
\hline & Netto et al. (2013) & Teste de evocação de Buschke & 1 & 1,9 \\
\hline & Netto et al. (2013) & Neupsilin & 1 & 1,9 \\
\hline & Santos et al. (2021) & Teste do Relógio & 1 & 1,9 \\
\hline & Santos et al. (2021) & Teste dos cinco dígitos & 1 & 1,9 \\
\hline \multirow{5}{*}{$\begin{array}{l}\text { Aspectos sociais } \\
\qquad(n=8)\end{array}$} & $\begin{array}{l}\text { Bastos et al. (2016); Inouye et al. (2010); } \\
\text { Reis et al. (2011) }\end{array}$ & Inventario de Percepção de Suporte Familiar (IPSF) & 3 & 5,9 \\
\hline & $\begin{array}{l}\text { Carneiro e Falcone (2016); Domenico- } \\
\text { Grazziotin e Scortegagna (2016) }\end{array}$ & Inventário de Habilidades Sociais (IHS) & 2 & 3,9 \\
\hline & Baptista et al. (2006) & Questionário de atividades sociais & 1 & 1,9 \\
\hline & Aparatto Júnior (2010) & Health Assessment Questionnaire (HAQ) & 1 & 1,9 \\
\hline & Carneiro e Falcone (2016) & Questionário de Dificuldades em Situações Sociais & 1 & 1,9 \\
\hline
\end{tabular}




\begin{tabular}{|c|c|c|c|c|}
\hline Construto avaliado & Autores & Instrumento & $n$ & $\%$ \\
\hline \multirow{8}{*}{$\begin{array}{l}\text { Qualidade de vida } \\
\qquad(n=12)\end{array}$} & $\begin{array}{l}\text { Chaves e Gil (2015); Meirelles et al. (2010); } \\
\text { Santos et al. (2013); Takemoto et al. (2011) }\end{array}$ & WHOQOL Brief & 4 & 7,8 \\
\hline & Guedes et al. (2013) & Nottingham Health Profile & 1 & 1,9 \\
\hline & Ramos et al. (2012) & Aging Perceptions Questionnaire (APQ) & 1 & 1,9 \\
\hline & Lima e Portela (2010) & Short-Form Health Survey & 1 & 1,9 \\
\hline & Guedes et al. (2013) & Sickness Impact Profile & 1 & 1,9 \\
\hline & Guedes et al. (2013); Inouye et al. (2010) & Escala Qualidade de Vida (Qdv-DA) & 2 & 3,9 \\
\hline & Lima e Portela (2010) & $\begin{array}{l}\text { Perfil de Qualidade de Vida Relacionada à Saúde de } \\
\text { Idosos Independentes (QUASI) }\end{array}$ & 1 & 1,9 \\
\hline & Chaves e Gil (2015) & WHOQOL SRPB & 1 & 1,9 \\
\hline \multirow{2}{*}{$\begin{array}{l}\text { Atividades de vida } \\
\quad \text { diária }(n=2)\end{array}$} & Lampert e Scortegagna (2017) & Índice de Katz (ABVD) & 1 & 1,9 \\
\hline & Gratão et al. (2019) & Instrumental activities of daily living scale & 1 & 1,9 \\
\hline Coping $(n=1)$ & Souza et al. (2007) & Inventário de Coping de Jalowiec & 1 & 1,9 \\
\hline Resiliência $(n=1)$ & Neves et al. (2015) & Resilience Scale & 1 & 1,9 \\
\hline Estresse $(n=1)$ & Oliveira et al. (2019) & Escala de Estresse Percebido & 1 & 1,9 \\
\hline Autoestima $(n=1)$ & Meurer et al. (2012) & Escala de Autoestima de Rosenberg & 1 & 1,9 \\
\hline
\end{tabular}

Nota. Elaboração dos autores.

Importante número de estudos se voltou à investigação de aspectos sociais $(9,3 \%)$, tais como suporte familiar, habilidades sociais, saúde, atividades sociais. Por fim, sete outros aspectos também foram avaliados por meio de instrumentos padronizados, mas de forma menos intensa: autopercepção, criatividade, ansiedade, coping, resiliência, estresse, autoestima e atividades de vida diária.

Também foram relatados o uso de outros métodos, tais como entrevistas $(n=3)$, questionário não especificado $(n=1)$ e Torre de Hanói, sem que maiores especificações sobre eles fossem fornecidas nos estudos. Uma hipótese a ser levantada envolve o fato de que pesquisadores de diversas outras áreas de conhecimento estiveram envolvidos nas pesquisas consultadas, de modo que o emprego desses outros métodos se justifica perante a restrição do uso dos testes psicológicos.

Em relação aos instrumentos, faz-se notar que o mais utilizado é o Mini Mental Exame Mental, sendo importante destacar que sua predominância se deve ao fato de que este instrumento foi amplamente utilizado como método avaliativo para inclusão ou exclusão dos participantes nas pesquisas. Constitui-se em um teste de rastreio de uso não privativo para psicólogos validado para população idosa e aprovado pelo SATEPSI. Este instrumento visa identificar possíveis alterações cognitivas, como a memória. Assim, a grande utilização do MEEM como instrumento de rastreio em pesquisas com idosos se justifica, pois além de ser de fácil e breve aplicação pode ser utilizado por qualquer profissional e é capaz de sugerir possíveis alterações cognitivas (Ministério da Saúde, 2006). Diversas pesquisas encontradas com essa população alvo acentuam as características negativas associadas ao envelhecimento, como o declínio cognitivo e o acometimento por doenças como o Alzheimer.
É importante citar que, dentre os instrumentos utilizados, a maior parte deles não é específica para a população idosa, sendo esse o caso do WAIS-III, cuja utilização pode se dar na faixa etária entre 16 a 89 anos, não sendo, portanto, um instrumento específico para a população idosa. A exceção ocorre em relação ao Teste Wisconsin de Classificação de Cartas (WCST), considerado um teste padrão ouro validado internacionalmente para população idosa com idade entre 60 e 89 anos (Silva-Filho et al., 2013), que tem por objetivo avaliar as funções executivas, apresentando um escore global sobre a capacidade de raciocínio abstrato, análise de comportamentos de perseverança, dificuldades de atenção na tarefa e déficit de aprendizagem, possíveis alterações ou lesões do lobo frontal além de avaliar da habilidade de modificação de estratégias cognitivas (Silva-Filho et al, 2013).

Outra importante constatação se refere à utilização de uma série de instrumentos breves ou de rastreio, cuja utilização nos trabalhos pode ser justificada perante a facilidade de aplicação, rapidez na obtenção de resultados e facilidade na interpretação dos dados. Esse tipo de instrumento vem sendo utilizado em indivíduos que estão aparentemente bem, mas que apresentam, por exemplo, um fator de risco para determinado quadro. Nesse sentido, o rastreio tem sido considerado perante a premissa de que o diagnóstico precoce pode contribuir para a melhora do prognóstico (Goulart \& Chiari, 2007).

Por fim, em menor proporção, foram identificados testes que mensuram construtos englobados pela Psicologia Positiva, sendo eles relacionados à qualidade de vida, autoestima, resiliência, coping e criatividade, de modo a apontar a escassez de trabalhos em avaliação psicológica que abordem aspectos positivos do indivíduo, tal como ressaltado por Pires et al. (2015) e Pureza et al. (2012), a partir de revisões da literatura. 


\section{M*' INTERACÃO EM LF PSICOLOGIA}

Assim, se faz notar que a amplitude de instrumentos para avaliação de declínio cognitivo, e outras perdas e doenças que são características do envelhecimento, se sobressai em relação aos instrumentos que visam a identificação de forças e virtudes pessoais, fato que pode ser justificado, em partes, pela tradição da Psicologia Clássica e das Políticas de Saúde, as quais, historicamente, se debruçaram sobre as patologias, doenças, incapacidades e limitações humanas, especialmente na velhice (Paludo \& Koller, 2007).

\section{CONSIDERAÇÕES FINAIS}

O número reduzido de publicações que abordam a Avaliação Psicológica aplicada ao idoso, em especial que abordem construtos da Psicologia Positiva, ressalta a necessidade de se expandir as pesquisas nessa fase desenvolvimental, Dentro desse cenário, mostra-se essencial que as investigações se voltem à avaliação e identificação, nos idosos, de suas forças e potencialidades, considerando-se principalmente o fato de que a tendência é que a população idosa seja predominante no Brasil dentro de alguns anos.

De modo geral, o número reduzido de estudos encontrados, assim como o número ainda restrito de instrumentos específicos para uso em idosos, reforçam a constatação de que a área da avaliação psicológica deve se atentar a essa população. Dentre as possibilidades, esforços voltados tanto ao desenvolvimento de instrumentos específicos para essa faixa etária, assim como a adaptação de instrumentos internacionais disponíveis em outros contextos, que considerem as particularidades dessa fase, se mostram essenciais. Em especial considerando-se os possíveis comprometimentos relacionados ao funcionamento cognitivo e sensorial (Edelstein et al., 2007). Somente desse modo uma avaliação adequada e fidedigna poderá ser conduzida junto a essa população.

Como limitação do estudo pode ser citado o número de bases de dados utilizadas, bem como o fato de que, a consulta às bases de dados foi feita nas suas versões eletrônicas, de modo que tal formato acaba por restringir o número de artigos disponibilizados. Consequentemente, algumas bases não contemplam artigos mais antigos, os quais, nem sempre se encontram disponibilizados na íntegra. Devido a essa questão, vários estudos podem não ter sido incluídos na revisão, de modo que os resultados aqui apresentados devem ser interpretados com cautela.

A seleção das palavras-chave e a impossibilidade de acesso a alguns textos completos também pode ter limitado os achados. Como sugestão de estudos futuros, sugere-se a ampliação das bases de dados, ampliando a pesquisa para o contexto internacional, assim como a realização da busca manual de artigos que abordem a temática.
Frente as contribuições que podem ser geradas a partir de pesquisas voltadas a esse público, notadamente aquelas com foco preventivo, voltadas à promoção da saúde, dentro de uma visão de envelhecimento bem-sucedido, o investimento na temática se mostra essencial. Ao conhecer o perfil das pesquisas que vêm sendo conduzidas, tais dados poderão ser utilizados na elaboração de políticas públicas, programas de intervenção e promoção de saúde, bem como atuar, de forma indireta, para a implantação de medidas voltadas à redução dos gastos públicos com saúde, de modo que sua relevância se justifica.

\section{DECLARAÇÃO DA CONTRIBUIÇÃO DOS AUTORES}

Certificamos que todos os autores participaram suficientemente do trabalho para tornar pública sua responsabilidade pelo conteúdo. A contribuição de cada autor pode ser atribuída como se segue: J.C. e T.C.N contribuíram para o desenvolvimento da ideia do artigo, bem como atuaram em todas as fases durante seu desenvolvimento. São responsáveis pela redação final (revisão e edição).

\section{DECLARAÇÃO DE CONFLITOS DE INTERESSE}

Os autores declaram que não há conflitos de interesse no manuscrito submetido.

\section{DECLARAÇÃO DE FINANCIAMENTO}

A pesquisa relatada no manuscrito foi financiada pela bolsa de mestrado da primeira autora (CNPq, proc. 131318/2019-5).

\section{REFERÊNCIAS}

Alberte, J. S. P., Ruscalleda, R. M. I., \& Guariento, M. E. (2015). Qualidade de vida e variáveis associadas ao envelhecimento patológico. Sociedade Brasileira de Clínica Médica, 13(1), 32-39. http://files.bvs.br/upload/S/1679-1010/2015/ v13n1/a4766.pdf

*Apratto Júnior, P. C. (2010). A violência doméstica contra idosos nas áreas de abrangência do Programa Saúde da Família de Niterói (RJ, Brasil). Ciência \& Saúde Coletiva, 15(6), 2983-2995. http://doi.org/10.1590/S141381232010000600037

Argimon, I. I. L. (2006). Aspectos cognitivos em idosos. Avaliação Psicológica, 5(2), 243-245. http://pepsic.bvsalud.org/ pdf/avp/v5n2/v5n2a15.pdf

Azeredo, Z. A. S., \& Afonso, M. A. N. (2016). Solidão na perspectiva do idoso. Revista Brasileira de Geriatria e Gerontologia, 19(2), 313-324. http://doi.org/10.1590/180998232016019.150085 


\section{H*' INTERACÃO EM LFICOLOGIA}

*Baptista, M. N., Morais, P. R., Rodrigues, T.,\& Silva, J. A. C. (2006). Correlação entre sintomatologia depressiva e prática de atividades sociais em idosos. Avaliação Psicológica, 5(1), 77-85. http://pepsic.bvsalud.org/scielo.php?script=sci_ arttext\&pid=S1677-04712006000100009\&lng=pt\&tlng=pt

Bartholomaeus, J. D., Agteren, J. E. M. V. Iasiello, M. P., Jarden, A., \& Kelly, D. (2019). Positive Aging: The Impact of a Community Wellbeing and Resilience Program. Clinical Gerontologist, 42(4), 377-386. http://doi.org/10.1080/073 17115.2018.1561582

*Bastos, D. S., Scortegagna, S. A., Baptista, M. N., \& Cremasco, G. S. (2016). Sintomas depressivos e suporte familiar em adultos em hemodiálise. Revista Psicologia: Teoria e Prática, 18(2), 103-116. http://doi.org/10.15348/1980-6906/ psicologia.v18n2p103-116

Bellingtier, J. A., \& Neupert, S. D. (2018). Negative aging attitudes predict greater reactive daily stressors in older adults. Journals of Gerontology: Psychological Sciences, 73(7), 1155-1159. http://doi.org/10.1093/geronb/gbw086

*Brito, I. L., Areosa, S. C., Lopes, R. M. F., \& Argimon, I. I. L. (2012). Avaliação das funções executivas em idosos acometidos por doenças crônico-degenerativas. Cuadernos de Neuropsicología, 6(1), 46-62. http://doi.org/10.7714/ cnps/6.1.203

*Carneiro, R. S., \& Falcone, E. (2016). Avaliação de um programa de promoção de habilidades sociais para idosos. Análise Psicológica, 34(3), 279-291. http://doi.org/10.14417/ap.960

Cavalcante, R. M. F., Dantas, D.S., Araújo, D. N., Magalhães, P. A. F., \& Neves, M. T. S. (2015). Contribuições de um grupo de terceira idade para a saúde de idosas participantes. $R e$ vista Brasileira de Ciências da Saúde, 19 (1), 11-18. http:// doi.org/10.4034/RBCS.2015.19.01.02

Cecato, J. F., Martinelli, J. E., Bartholomeu, L. L., Ferreira, P. P., Montiel, J. M., \& Bartholomeu, D. (2013). Correlações entre aspectos da memória e aprendizagem em população idosa. Revista de Ciências Humanas, 47(1), 133-151. http:// dx.doi.org/10.5007/2178-4582.2013v47n1p133

*Chaves, L. J., \& Gil, C. A. (2015). Older people's concepts of spirituality, related to aging and quality of life. Ciência \& Saúde Coletiva, 20(12), 3641-3652. http://doi.org/10.1590/1413812320152012.19062014

Craciun, C., Gellert, P., \& Flick, U. (2017). Aging in precarious circumstances: do positive views on aging make a difference? The Gerontologist, 57(3), 517-528. http://doi. org/10.1093/geront/gnv135

Cupertino, A. P. F. B., Rosa, F. H. M., \& Ribeiro, P. C. C. (2007). Definição de envelhecimento saudável na perspectiva de indivíduos idosos. Psicologia Reflexão e Crítica, 20(1), 8186. http://doi.org/10.1590/S0102-79722007000100011
Dawalibi, N. W., Anacleto, G. M. C., Witter, C., Goulart, R. M. M., \& Aquino, R. C. (2013). Envelhecimento e qualidade de vida: análise da produção científica da SciELO. Estudos de Psicologia (Campinas), 30 (3), 393-403. http://doi. org/10.1590/S0103-166X2013000300009

Denmark, F. L., \& Zarbiv, T. (2016). Living Life to the Fullest: A Perspective on Positive Aging. Women \& Therapy, 39(3-4), 315321. http://doi.org/10.1080/02703149.2016.1116864

Docking, R. E., \& Stock, J. (2017). Introduction. In R. E. Docking \& J. Stock (Eds.), International Handbook of Positive Aging (pp. 3-4). Routledge.

*Domenico-Grazziotin, J. B., \& Scortegagna, S. A. (2016). Avaliação das habilidades sociais em adultos idosos e adultos. Temas em Psicologia, 24(2), 695-705. http://doi. org/10.9788/TP2016.2-16Pt

Edelstein, B. A., Woodhead, E. L., Segal, D. L., Heisel, M. J., Bower, E. H., Lowery, A. J., \& Stoner, S. A. (2007). Older Adult Psychological Assessment, Clinical Gerontologist, 31(3), 1-35. http://doi.org/10.1080/07317110802072108

Esteve-Clavero, A., Ayora-Folch, A., Maciá-Soler, L., \& Molés-Julio, M. P. (2018). Fatores associados à qualidade de vida dos idosos. Acta Paulista de Enfermagem, 31(5), 542549. http://doi.org/10.1590/1982-0194201800075

*Esteves, C. S., Oliveira, C. R., Irigaray, T. Q., \& Argimon, I. I. L. (2016). Desempenho de idosos com e sem sintomas depressivos no WCST-64. Avaliação Psicológica, 15(1), 31 39. http://doi.org/10.15689/1p.2016.1501.04

Fernández-Ballesteros, R. (2009). Envejecimento activo: contribuiciones de la Psicología. Ediciones Pirámide.

Finkenzeller, T., Pötzelsberger, B., Kösters, A., Würth, S., Amesberger, G., Dela, F., \& Müller, E. (2019). Aging in high functioning elderly persons: study design and analyses of behavioral and psychological factors. Scandinavian Journal of Medicine and Science Sports, 29(1), 7-16. http://doi. org/10.1111/sms. 13368

Fonseca, S. M. (2010). Promoção do desenvolvimento psicológico no envelhecimento. Contextos Clínicos, 3(2), 124131. http://doi.org/10.4013/ctc.2010.32.06

*Foss, M. P., Bastos-Formigheuri, M. S., \& Speciali, J. G. (2010). Figuras Complexas de Rey para idosos. Avaliação Psicológica, 9(1), 53-61. http://pepsic.bvsalud.org/scielo.php?script=sci_arttext\&pid=S1677-04712010000100007

Freire, G. V., Moura, W. B., Rocha, F. C. V., Madeiras, M. Z. A., \& Amorim, F. C. M. (2015). Perfil de idosos que frequentam um centro de convivência da terceira idade. Revista Interdisciplinar, 8(2), 11-19. https://revistainterdisciplinar.uninovafapi. edu.br/index.php/revinter/article/view/619/pdf_2 


\section{M." INTERACÃO EM PSICOLOGIA}

Goulart, B. N. G., \& Chiari, B. M. (2007). Testes de rastreamento $x$ testes de diagnóstico: atualidades no contexto da atuação fonoaudiológica. Pró-Fono Revista de Atualização Científica, 19(2), 223-232. http://www.scielo.br/pdf/pfono/ v19n2/a11v19n2.pdf

*Gratão, A. C. M., Brigola, A. G., Ottaviani, A. C., Luchesi, B. M., Souza, E. N.,... lost, S. C. (2019). Brief version of Zarit Burden Interview (ZBI) for burden assessment in older caregivers. Dementia \& Neurospychologia, 13(1), 122-129. http:// doi.org/10.1590/1980-75642018dn13-010015

*Guedes, D. V., Barbosa, A. J. G., \& Magalhães, N. C. (2013). Qualidade de vida de idosos com declínio cognitivo: auto e heterorrelatos. Avaliação Psicológica, 12(1), 9-17. http:// pepsic.bvsalud.org/scielo.php?script=sci_arttext\&pi$\mathrm{d}=$ S1677-04712013000100003\&lng=pt\&tlng=pt

*Gurgel, M. G. A., \& Sisto, F. F. (2010). Estudo correlacional entre inteligência e memória em idosos. Avaliação Psicológica, 9(2), 163-172. http://pepsic.bvsalud.org/pdf/avp/ v9n2/v9n2a03.pdf

Hughes J. C. (2016) The Physiology and Psychology of Aging: Should Aging Be Successful or Authentic?. In G. Scarre (Ed.), The Palgrave Handbook of the Philosophy of Aging (pp.49-68). Palgrave Macmillan.

Ingrand, I., Paccalin, M., Liuu, E., Gil, R., \& Ingrand, P. (2018). Positive perception of aging is a key predictor of quality-of-life in aging people. PLOS ONE 13(10), e0204044. http:// doi.org/10.1371/journal.pone.0204044

*Inouye, K., Barham, E. J., Pedrazzani, E. S., \& Pavarini, S. C. I. (2010). Percepções de suporte familiar e qualidade de vida entre idosos segundo a vulnerabilidade Social. Psicologia: Reflexão e Crítica, 23(3), 582-592. http://doi.org/10.1590/ S0102-79722010000300019

Kreuz, G., \& Franco, M. H. P. (2017). O luto do idoso diante das perdas da doença e do envelhecimento: revisão sistemática de literatura. Arquivos Brasileiros de Psicologia, 69(2), 168186. http://pepsic.bvsalud.org/scielo.php?script=sci_arttext\&pid=S1809-52672017000200012\&lng=pt\&tlng=pt

*Lampert, C. D. T., \& Scortegagna, S. A. (2017). Avaliação das condições de saúde e distorções cognitivas de idosos com depressão. Avaliação Psicológica, 16(1), 48-58. http://doi. org/10.15689/ap.2017.1601.06

Lampert, C. D. T., \& Scortegagna, S. A. (2019). Autopercepção no Rorschach-SC de idosos com depresssão emu ma perspectiva idiográfica. Psico, 50(3), 229884. http://doi. org/10.15448/1980-8623.2019.3.29884

Lima, A., Ramos, J., Bezerra, I., Rocha, R, Batista, H., \& Pinheiro, W. (2016). Depressão em idosos: uma revisão sistemática da literatura. Revista de Epidemiologia e Controle de Infecção, 6(2), 96-103. http://doi.org/10.17058/reci.v6i2.6427

Lima, A. M. M., Silva, H. S., \& Galhardoni, R. (2008). Envelhecimento bem-sucedido: trajetórias de um constructo e novas fronteiras. Interface - Comunicação, Saúde e Educação, 12(27), 795807. http://doi.org/10.1590/S1414-32832008000400010
Janaina Chnaider e Tatiana de Cássia Nakano
*Lima, M. J. B., \& Portela, M. C. (2010). Elaboração e avaliação da confiabilidade de um instrumento para medição da qualidade de vida relacionada à saúde de idosos independentes. Cadernos de Saúde Pública, 26(8), 1651-1662. http://doi.org/10.1590/S0102-311X2010000800018

Lopes, C. I. P., Aguinha, M. S., Lopes, F. D. O. C. F., Petronilho, S. M. S., Valeiro, M. P., \& Dixe, M. A. C. R. (2010). Esperança e desesperança nos idosos. International Journal of Developmental and Educational Psychology, 1(2), 883-894. redalyc. org/service/redalyc/downloadPdf/3498/349832325092/6

*Lopes, R. M. F., \& Argimon, I. I. L. (2009). Prejuízos Cognitivos em idosos com Diabetes Mellitus tipo 2. Cuadernos de Neuropsicología, 3(2), 171-197. http:// pepsic.bvsalud.org/scielo.php?script=sci_arttext\&pi$d=S 0718-41232009000200005 \&$ lng=pt\&tlng=es

*Meirelles, B. H. S., Arruda, C., Simon, E., Vieira, F. M. A., Cortezi, M. D. V., \& Natividade, M. S. L. (2010). Condições associadas à qualidade de vida dos idosos com doença crônica Cogitare Enfermagem, 15(3),433-440. http://dx.doi. org/10.5380/ce.v15i3

Mello, B. L. D., Haddad, M. C. L., \& Dellaroza, M. S. G. (2012). Avaliação cognitiva de idosos institucionalizados. Acta Scientiarium, 34(1), 95-102. http://doi.org/10.4025/actascihealthsci.v34i1.7974

*Meurer, S. T., Luft, C. B., Benedetti, T. R., \& Mazo, G. Z. (2012). Validade de construto e consistência interna da escala de autoestima de Rosenberg para uma população de idosos brasileiros praticantes de atividades físicas. Motricidade, 8(4), 5-15. http://doi.org/10.6063/motricidade.8(4).1548

Ministério da Saúde (2006). Envelhecimento e saúde da pessoa idosa. Secretaria de Atenção à Saúde, Departamento de Atenção Básica. http://bvsms.saude.gov.br/bvs/publicacoes/evelhecimento_saude_pessoa_idosa.pdf

Moher, D., Liberati, A., Tetzlaff, J., \& Altman, D. G. (2009). Preferred Reporting Items for Systematic Reviews and Meta- Analyses: The PRISMA Statement. PLoS Med, 6(7), e1000097. http://doi.org/10.1371/journal.pmed1000097

Moher, D., Shamseer, L., Clarke, M., Ghersi, D., Liberati, A., Petticrew, M., Shekelle, P., Stewart, L. A., \& Group, P. (2015). Preferred reporting items for systematic review and meta-analysis protocols (PRISMA-P) 2015 statement. Systematic reviews, 4(1), 1-9. https://doi.org/10.1186/2046-4053-4-1

*Netto, T. M., Greca, D. V., Zimmermann, N., Oliveira, C. R., Teixeira-Leite, H. M., Fonseca, R. P., \& Landeira-Fernandez, J. (2013). Efeito de um programa de treinamento da memória de trabalho em adultos idosos. Psicologia: Reflexão e Crítica, 26(1), 122-135. http://doi.org/10.1590/S010279722013000100014

*Neves, M., Martins, A. B., D'ávila, O. P., Hilgert, J. B, \& Hugo, F. N. (2015). Idosos com maior potencial de resiliência podem estar mais satisfeitos com os serviços odontológicos?. Revista de Odontologia da UNESP, 44(6), 326-334. http://doi.org/10.1590/1807-2577.07214 


\section{H MTERAC̄OOEM ET PSICOLOGIA}

*Oliveira, C. R., Lima, M. M. B. M. P., Esteves, C. S., Gonzatti, V., Viana, S. A. R., Irigaray, T. Q., \& Argimon, I. I. L. (2017). Normative data of the Brazilian elderly in Logical Memory subtest of WMS-R. Avaliação Psicológica, 16(1), 11-18. http:// doi.org/10.15689/ap.2017.1601.02

*Oliveira, N. A., Souza, E. N., Brigola, A. G., Rossetti, E. S., Terassi, M., Luchesi, B. M., Inouye, K., \& Pavarini, S. C. I. (2019). Idosos cuidadores em diferentes arranjos de moradia: comparação do perfil de saúde e de cuidado. Revista Gaúcha de Enfermagem, 40, e20180225. http://doi. org/10.1590/1983-1447.2019.20180225

Organização Mundial da Saúde (2005). Envelhecimento Ativo: uma política de saúde. OMS. http://bvsms.saude.gov.br/ bvs/publicacoes/envelhecimento_ativo.pdf

Pacico, J. C., \& Bastianello, M. R. (2014). As origens da Psicologia Positiva e os primeiros estudos brasileiros. In C. S. Hutz (Org.), Avaliação em Psicologia Positiva (pp. 13-22). Artmed.

Paludo, S. S., \& Koller, S. H. (2007). Psicologia positiva: Uma nova abordagem para antigas questões. Paidéia. 17(36), 9-20. http://doi.org/10.1590/S0103-863X2007000100002

Park, J., \& Hess, T. M. (2019): The effects of personality and aging attitudes on well-being in different life domains. Aging \& Mental Health. http://doi.org/10.1080/13607863. 2019.1660849

Paskulin, L. M. G., Córdova, F. P., Costa, F. M., \& Vianna, L. A. C. (2010). Percepção de pessoas idosas sobre qualidade de vida. Acta Paulista de Enfermagem, 23(1), 101-107. https:// doi.org/10.1590/S0103-21002010000100016

Pires, J. G., Nunes, M. F. O., \& Nunes, C. H. S. S. (2015). Instrumentos Baseados em Psicologia Positiva no Brasil: uma Revisão Sistemática. Psico-USF, 20 (2), 287-295. http:// doi.org/10.1590/1413-82712015200209

Pureza, J. R., Kuhn, C. H.C., Castro, E. K., \& Lisboa, C. S. M. (2012). Psicologia positiva no Brasil: uma revisão sistemática da literatura. Revista Brasileira de Terapias Cognitivas, 8(2), 109-117. http://doi.org/10.5935/18085687.20120016

*Ramos, L. M. B. C. Rocha, M., Gomes, I., \& Schwanke, C. H. A. (2012). Tradução e adaptação cultural do APQ - Questionário sobre percepções do envelhecimento para a língua portuguesa brasileira. Revista Brasileira de Geriatria e Gerontologia, 15 (2), 233-242. http://doi.org/10.1590/ S1809-98232012000200006

*Reis, L. A., Torres, G. V., Reis, L. A., Fernandes, M. H., \& Nobre, T. T. X. (2011). Avaliação do suporte familiar em idosos residentes em domicílio. Avaliação Psicológica, 10(2), 107115. http://pepsic.bvsalud.org/scielo.php?script=sci_arttext\&pid=S1677-04712011000200002\&lng=pt\&tlng=pt

Ribeiro, P. C. C. (2015). A psicologia frente aos desafios do envelhecimento populacional. Gerais: Revista Interinstitucional de Psicologia, 8(spe), 269-283. http:// pepsic.bvsalud.org/scielo.php?script $=$ sci_arttext\&pi$d=S 1983-82202015000200009 \&$ lng $=p t \&$ tlng=pt
Janaina Chnaider e Tatiana de Cássia Nakano
*Rien, M., Scortegagna, S. A., Grazziotin, J. B. D., \& Bertolin, T. E. (2017). Validity evidence of the Zulliger-CS in older adults with Parkinson's disease. Estudos de Psicologia (Campinas), 34(4), 560-570. http://doi.org/10.1590/198202752017000400011

*Santos, C. L. S., Alves, C. R., Alves, M. R., Teles, M. A. B., \& Fonseca, J. R. (2012). Autopercepção dos idosos sobre satisfação com a vida relacionada a interações sociais e lazer. Motricidade, 8 (2), 232-239. https://www.redalyc. org/pdf/2730/273023568029.pdf

*Santos, C. M., Hugo, F. N., Leal, A. F., \& Hilgert, J. B. (2013). Comparison of two assessments instruments of quality of life in older adults. Revista Brasileira de Epidemiologia, 16(2), 328-337. http://doi.org/10.1590/S1415$790 \times 2013000200009$

*Santos, M. R., Krug, M. S., Brandão, M. R., Leon, V. S., Martinotto, J. C., Fonseca, J. D., Brasil, A. C., Machado, A. G., Oliveira, A. A. (2021). Effects of musical improvisation as a cognitive and motor intervention for the elderly. Estudos de Psicologia (Campinas), 38, e190132. http://doi.or$\mathrm{g} / 10.1590 / 1982-0275202138 \mathrm{e} 190132$

Schneider, R. H., \& Irigaray, T. Q. (2008). O envelhecimento na atualidade: aspectos cronológicos, biológicos, psicológicos e sociais. Estudos de Psicologia (Campinas), 25(4), 585593. http://doi.org/10.1590/S0103-166X2008000400013

Scorsolini-Comin, F., \& Santos, M. A. (2010). Psicologia positiva e os instrumentos de avaliação no contexto brasileiro. Psicologia: Reflexão e Crítica, 23(3), 440-448. http://doi. org/10.1590/S0102-79722010000300004

*Silva-Filho, J. H., Pasian, S. R., \& Vale, F. A. C. (2007). Typical performance of elderly patients with Alzheimer disease on the Wisconsin Card Sorting Test (WCST). Dementia \& Neuropsychologia, 1(2), 181-189. http://doi.org/10.1590/ s1980-57642008dn10200011

*Soares, R. M., Diniz, A. B., \& Cattuzzo, M. T. (2013). Associação entre atividade física, aptidão física e desempenho cognitivo em idosos. Motricidade, 9(2), 84-93. http://doi. org/10.6063/motricidade.9(2).2670

*Souza, A. A. F., \& Wechsler, S. M. (2013). Inteligência e criatividade na maturidade e velhice. Psicologia: Reflexão e Crítica, 26(4), 643-653. http://doi.org/10.1590/S010279722013000400004

*Souza, J. N., Chaves, E. C., \& Caramelli, P. (2007). Coping em idosos com doença de Alzheimer. Revista Latino-Americana de Enfermagem, 15(1), 93-99. http://doi.org/10.1590/ S0104-11692007000100014

*Takemoto, A. Y., Okubo, P., Bedendo, J., \& Carreira, L. (2011). Avaliação da qualidade de vida em idosos submetidos ao tratamento hemodialítico. Revista Gaúcha de Enfermagem, 32(2), 256-262. http://doi.org/10.1590/S198314472011000200007 
Teixeira, I. N. A., \& Neri, A. L. (2008). Envelhecimento bem-sucedido: uma meta no curso da vida. Psicologia USP, 19(1), 8194. https://doi.org/10.1590/S0103-65642008000100010

Veras, R. P. (2012). Prevenção de doenças em idosos: os equívocos dos atuais modelos. Cadernos de Saúde Pública, 28(10), 1834-1840. http://doi.org/10.1590/S0102$311 \times 2012001000003$
Vieira, R. S., \& Lima M. E. O. (2015). Estereótipos sobre os Idosos: Dissociação entre Crenças Pessoais e Coletivas. Temas em Psicologia, 23(4), 947-958. http://doi. org/10.9788/TP2015.4-11

Data de submissão: 03/03/2020

Primeira decisão editorial: 29/06/2020

Aceite em 01/09/2020 